\title{
Chung-Feller Property in View of Generating Functions
}

\author{
Shu-Chung Liu * \\ Department of Applied Mathematics \\ National Hsinchu University of Education \\ Hsinchu City, Taiwan \\ liularry@mail.nhcue.edu.tw \\ Yi Wang ${ }^{\dagger}$ \\ Department of Applied Mathematics \\ Dalian University of Technology \\ Dalian, China \\ wangyi@dlut.edu.cn \\ Yeong-Nan Yeh $\ddagger \S$ \\ Institute of Mathematics \\ Academia Sinica \\ Taipei, Taiwan \\ mayeh@math.sinica.edu.tw
}

Submitted: Aug 16, 2009; Accepted: Apr 29, 2011; Published: May 8, 2011

Mathematics Subject Classification: 05A15, 05A18

\begin{abstract}
The classical Chung-Feller Theorem offers an elegant perspective for enumerating the Catalan number $c_{n}=\frac{1}{n+1}\left(\begin{array}{c}2 n \\ n\end{array}\right)$. One of the various proofs is by the uniformpartition method. The method shows that the set of the free Dyck $n$-paths, which have $\left(\begin{array}{c}2 n \\ n\end{array}\right)$ in total, is uniformly partitioned into $n+1$ blocks, and the ordinary Dyck $n$-paths form one of these blocks; therefore the cardinality of each block is $\frac{1}{n+1}\left(\begin{array}{c}2 n \\ n\end{array}\right)$. In this article, we study the Chung-Feller property: a sup-structure set can be uniformly partitioned such that one of the partition blocks is (isomorphic to) a well-known structure set. The previous works about the uniform-partition method used bijections, but here we apply generating functions as a new approach. By claiming a functional equation involving the generating functions of sup- and sub-structure sets, we re-prove two known results about Chung-Feller property, and explore several new examples including the ones for the large and the little Schröder paths. Especially for the Schröder paths, we are led by the new approach straightforwardly to consider "weighted" free Schröder paths as sup-structures. The weighted structures are not obvious via bijections or other methods.
\end{abstract}

\footnotetext{
*Partially supported by NSC 98-2115-M-134-005-MY3

${ }^{\dagger}$ Partially supported by NSFC 11071030

${ }^{\ddagger}$ Partially supported by NSC 98-2115-M-001-019-MY3

${ }^{\S}$ Corresponding author
} 


\section{Introduction}

We use $\mathbb{Z}, \mathbb{N}$ and $\mathbb{N}^{-}$to denote the sets of integers, natural numbers and non-positive integers, respectively. The combinatorial structures discussed in the paper are lattice paths (or random walks) that start at the origin $(0,0)$ and lie in $\mathbb{N} \times \mathbb{Z}$ or $\mathbb{N} \times \mathbb{N}$. A class of lattice paths is usually determined by a step set $S$ consisting of finite-many (fundamental) steps, and a step is an integral vector $(a, b)$ with $a \geq 1$. We call $(a, b)$ an rise step if $b>0$, fall step if $b<0$, and level step if $b=0$. Given a lattice path $P$, let $\ell(P)$ denote the length of $P$, which is the $x$-coordinate of the right end point of $P$ but not necessarily the number of steps on $P$.

Let $\mathcal{L}_{n, S}, \mathcal{L}_{n, S}^{+}$and $\mathcal{L}_{n, S}^{-}$be the sets of lattice paths from $(0,0)$ to $(n, 0)$ that are constructed by steps in $S$ and lie respectively in $\mathbb{N} \times \mathbb{Z}, \mathbb{N} \times \mathbb{N}$ and $\mathbb{N} \times \mathbb{N}^{-}$. By reversing the order of the steps in each path, we obtain a bijection between $\mathcal{L}_{n, S}^{+}$and $\mathcal{L}_{n, S}^{-}$. Sometimes we focus on the lattice paths with end point $(n, h)$ for fixed positive integers $h$. We use $\mathcal{L}_{(n, h), S}$ and $\mathcal{L}_{(n, h), S}^{+}$to denote the sets of such lattice paths. The paths of all lengths are often discussed at a time, especially when we deal with their generating function (GF); so we define the class $\mathcal{L}_{\mathbb{N}, S}:=\bigcup_{n \geq 0} \mathcal{L}_{n, S}, \mathcal{L}_{k \mathbb{N}, S}:=\bigcup_{n \geq 0} \mathcal{L}_{k n, S}, \mathcal{L}_{(\mathbb{N}, h), S}:=\bigcup_{n \geq 0} \mathcal{L}_{(n, h), S}$ and so on.

A lattice path is called a flaw path if it have some steps below or partially below the $x$-axis, which are called flaw steps; because the paths without flaws were once named "good" in the literature and draw more attention. The paths in $\mathcal{L}_{n, S}$ are called free paths, because they do not face the boundary $x$-axis as the ones in $\mathcal{L}_{n, S}^{+}$

Let us recall the original the Chung-Feller theorem and its known generalization as follows. The Catalan number $c_{n}=\frac{1}{n+1}\left(\begin{array}{c}2 n \\ n\end{array}\right)$ is one of the most investigated sequences. Among hundred of known combinatorial structures interpreting $c_{n}[28,29]$, the Dyck $n$ path is well known and fascinating. Usually, the set of all Dyck $n$-paths is denoted by $\mathcal{D}_{n}$, which is actually $\mathcal{L}_{2 n, S_{\mathcal{D}}}^{+}$with $S_{\mathcal{D}}=\{U=(1,1), D=(1,-1)\}$. Dyck paths are 2dimensional translations of Dyck language, named after Walther von Dyck, which consists of all balanced strings of parentheses. As random walks, Dyck paths also visually interpret the tight-match version of the ballot problem. The original ballot problem deals with a dominant-match, and was introduced and proved inductively by Bertrand [3]. The reader can refer to Renault's interesting narratives $[22,23]$ about the ballot problem. Especially, he recovered André's actual method for solving the classical ballot theorem and rectified the prevalence of mis-attribution.

Given a positive integer $d$, let $S_{\mathcal{C}^{(d)}}=\left\{U=(1,1), D_{d}=(1,-d)\right\}$ and

$$
\mathcal{C}_{n}^{(d)}=\mathcal{L}_{(d+1) n, S_{\mathcal{C}^{(d)}}}^{+}
$$

The elements of $\mathcal{C}_{n}^{(d)}$ are called the Catalan n-paths of order $d$. Catalan paths are generalized from Dyck paths, and $\mathcal{C}_{n}^{(1)}=\mathcal{D}_{n}$. Notice that the index $n$ is the semi-length of the paths in $\mathcal{D}_{n}$, and $n$ is $\frac{1}{d+1}$ of the length of each path in $\mathcal{C}_{n}^{(d)}$. It is known that $c_{n}^{(d)}:=\left|\mathcal{C}_{n}^{(d)}\right|=\frac{1}{d n+1}\left(\begin{array}{c}(d+1) n \\ n\end{array}\right)$, which is called the $n$th generalized Catalan number $[13,15]$.

Given $0 \leq k \leq d n$, an $(n, k)$-flaw path is a path in $\mathcal{L}_{(d+1) n, S_{\mathcal{C}^{(d)}}}$ that contains $k$ rise 
steps $U$ below the $x$-axis. Let $\mathcal{C}_{n, k}^{(d)}$ be the set of all $(n, k)$-flaw paths. Clearly, $\mathcal{C}_{n, d n}^{(d)} \cong$ $\mathcal{C}_{n, 0}^{(d)}=\mathcal{C}_{n}^{(d)}$. Not only $\mathcal{L}_{(d+1) n, S_{\mathcal{C}^{(d)}}}$ is the disjoint union of $\left\{\mathcal{C}_{n, k}^{(d)}\right\}_{k=0}^{d n}$, but a stronger property was developed as follows:

Theorem 1.1 [11] The structure set $\mathcal{L}_{(d+1) n, S_{\mathcal{C}^{(d)}}}$ is partitioned uniformly into $\left\{\mathcal{C}_{n, k}^{(d)}\right\}_{k=0}^{d n}$. Therefore, for each $k$ we have

$$
\left|\mathcal{C}_{n, k}^{(d)}\right|=\frac{1}{d n+1}\left|\mathcal{L}_{(d+1) n, S_{\mathcal{C}^{(d)}}}\right|=c_{n}^{(d)}
$$

The above theorem was first proved by Eu et al. recently [11]. They used the cut-and-paste technique to derive a bijection between $\mathcal{C}_{n}^{(d)}$ and $\mathcal{C}_{n, k}^{(d)}$. As a relative result of Theorem 1.1, a generalized ballot problem with step set $S_{\mathcal{C}^{(d)}}$ was proved by Renault [23] using André's "actual" method.

In particular, Eq. (1) confirms $\left|\mathcal{D}_{n}\right|=\frac{1}{n+1}\left(\begin{array}{c}2 n \\ n\end{array}\right)$ with $d=1$. There are many other proof methods for the identity $\left|\mathcal{D}_{n}\right|=\frac{1}{n+1}\left(\begin{array}{c}2 n \\ n\end{array}\right)$, including the Cycle Lemma, the reflection method, the counting of permutations, etc. The method using uniform partition is particularly called the Chung-Feller Theorem, which was first proved by MacMahon [18] and then re-proved by Chung and Feller [8]. Some other interesting proofs and generalizations are given in $[4,5,6,9,12,21,27,31]$.

The well-known Motzkin paths admit a Chung-Feller type result too. This problem was first noted by Shapiro in [26], where the extension and the partition blocks were suggested by an anonymous referee. Shapiro also mentioned that this property can be proved either by the Cycle Lemma or by the generating function. A proof using bijection and uniform partition was given by Eu et al. [11]. We will fulfill a proof using generating functions in the next section and discuss Chung-Feller type results for generalized ( $k$-color) Motzkin paths in Section 3.

Our interest is less in the cardinality but more in Chung-Feller type results, i.e., the phenomenon that a sup-structure set, like $\mathcal{L}_{(d+1) n, S_{\mathcal{D}}}$, can be partitioned uniformly, and one of these partition blocks is isomorphic to a well-known sub-structure set, like $\mathcal{D}_{n}$. (So is every block.) We call this phenomenon the Chung-Feller property admitted by the sub-structures (or the set of these sub-structures), and call the sup-structure set a Chung-Feller extension. Briefly, we will use "CF" to stand for "Chung-Feller".

The core of Chung-Feller type results is uniform partition. All previous known results are proved via bijection, i.e., showing the isomorphism among all partition blocks. These bijections are very sophisticated; however, each one is case by case without a general rule. Here we would like introduce a much easy and general way via generating functions to fulfill the idea of uniform partition.

The paper is organized as follows. In Section 2, we develop the proper generating function to deal with the CF extension of a given sub-structure. Then we re-prove the known CF-property for Catalan paths and Motzkin paths. In Section 3, we focus on multivariate generating functions for the discussing sub-structures. By this way, we discover a CF property for the generalized ( $k$-colored) Motzkin paths of order $d$. A various CF property for the Dyck paths with extension rate $2 n+1$ turns to be a special case. In 
Sections 4 and 5, we explore the CF property of the large and the little Schröder paths, respectively.

\section{The generating function of a CF extension}

The main purpose of this paper is to study the Chung-Feller property via generating functions. It is easy to derive the generating function of a CF extension (a set of supstructures) if it exists. By manipulating this generating function, we can explain what would these sup-structures look like.

For explaining our new approach, let us consider the classical Chung-Feller Theorem as an example. The set $\mathcal{D}_{n}:=\mathcal{L}_{2 n, S_{\mathcal{D}}}^{+}$of Dyck paths, where $S_{\mathcal{D}}=\{U=(1,1), D=(1,-1)\}$, is the discussing sub-structure set. The generating function of $\mathcal{D}_{\mathbb{N}}$ is $C(x)=\sum_{n \geq 0} c_{n} x^{n}$, where $c_{n}=\frac{1}{n+1}\left(\begin{array}{c}2 n \\ n\end{array}\right)$ is the $n$ th-Catalan number. The sup-structure set in this case is $\mathcal{L}_{2 n, S_{\mathcal{D}}}$, which can be partition uniformly into $\left\{\mathcal{C}_{n, k}\right\}_{k=0}^{n}$, where $k$ counts the the number of rise steps $U$ below the $x$-axis. (Particularly, $\mathcal{C}_{n, 0}=\mathcal{D}_{n}$.) It is natural to consider the following bivariate generating function for the sup-structure set $\mathcal{L}_{2 n, S_{\mathcal{M}}}$ according to its partitions.

$$
\begin{aligned}
\sum_{n \geq 0} \sum_{k=0}^{n}\left|\mathcal{C}_{n, k}\right| x^{n} y^{k} & =\sum_{n \geq 0} c_{n} x^{n}\left(1+y+\cdots+y^{n}\right) \\
& =\sum_{n \geq 0} c_{n} x^{n} \frac{1-y^{n+1}}{1-y} \\
& =\frac{C(x)-y C(x y)}{1-y} .
\end{aligned}
$$

No doubt that a CF extension of any sub-structure set has the same type of bivariate generating functions as (2). Therefore, we are led to the following definition:

Definition 2.1 Suppose $G(x):=\sum_{n>0} s_{n} x^{n}$ is the generating function of a class $\mathcal{S}_{\mathbb{N}}:=$ $\biguplus_{n \geq 0} \mathcal{S}_{n}$ of combinatorial structures with $s_{n}=\left|\mathcal{S}_{n}\right|$ and let $A_{G}(x)=x G(x)$. The generating function of Chung-Feller extension with respect to $G(x)$ (or with respect to the class $\left.\mathcal{S}_{\mathbb{N}}\right)$ is denoted and defined by

$$
\mathrm{CF}_{G}(x, y):=\frac{G(x)-y G(x y)}{1-y}=\left.\frac{A_{G}(x)-A_{G}(z)}{x-z}\right|_{z=x y} .
$$

By this definition we can easily reprove the classical Chung-Feller Theorem as follows. A short proof for the classical Chung-Feller Theorem Let $C(x)$ be the generating function of $\mathcal{D}_{\mathbb{N}}$ and $A(x)=x C(x)$. Clearly, $A(x)$ is the GF of lifted Dyck paths, i.e., the paths of form $U$-a Dyck path- $D$. It is well know that $C=1+x C^{2}$ or $1=C-x C^{2}$. So $x=A(x)-[A(x)]^{2}$. Plugging this identity into (3), we get

$$
\mathrm{CF}_{C}(x, y)=\left.\frac{A(x)-A(z)}{A(x)-[A(x)]^{2}-A(z)+[A(z)]^{2}}\right|_{z=x y}=\frac{1}{1-[A(x)+A(x y)]} .
$$


Obviously, this is the bivariate GF of the free Dyck paths where the exponent of $y$ counts the semi-length of the flaw steps on each path. Therefore, the free Dyck paths is a ChungFeller extension of Dyck paths and the cardinality, $\left(\begin{array}{c}2 n \\ n\end{array}\right)$, of the free Dyck $n$-paths is then $n+1$ times the cardinality of $\mathcal{D}_{n}$.

Following the definition (3) and reversing the process in (2), we obtain

$$
\mathrm{CF}_{G}(x, y)=\sum_{n \geq 0} \sum_{k=0}^{n} s_{n} x^{n} y^{k} \quad \text { and } \quad \mathrm{CF}_{G}(x, 1)=\sum_{n \geq 0}(n+1) s_{n} x^{n} .
$$

The second identity indicates that the extension rate is $n+1$. If we can find a supstructure class $\mathcal{E}_{\mathbb{N}}=\biguplus_{n \geq 0} \mathcal{E}_{n}$ to realize the generating $\mathrm{CF}_{G}(x, 1)$ as well as a collection of sub-structure sets $\left\{\mathcal{E}_{n, k}\right\}_{k=0}^{n}$ of $\mathcal{E}_{n}$ to realize the generating $\mathrm{CF}_{G}(x, y)$, then $\left\{\mathcal{E}_{n, k}\right\}_{k=0}^{n}$ forms a uniform $(n+1)$-partition of $\mathcal{E}_{n}$ and $\left|\mathcal{E}_{n, k}\right|=s_{n}$ for every $k$. If we can go a step further to have $\mathcal{E}_{n, k} \cong \mathcal{S}_{n}$ for some $k$, then the structure class $\mathcal{S}_{\mathbb{N}}$ admits the CF property and $\mathcal{E}_{\mathbb{N}}$ is its $\mathrm{CF}$ extension.

Indeed, $\mathrm{CF}_{G}(x, 1)$ is simply the first derivative of $x G(x)$, and it seems that we can directly reveal CF property by investigating $x G(x)$. As a matter of fact, it is difficult to interpret sup-structures only using $\mathrm{CF}_{G}(x, 1)$ or $x G(x)$. However, it turns easier after we explore the meaning of $y$ in $\mathrm{CF}_{G}(x, y)$.

The previous uniform partition proof of Chung-Feller Theorem uses bijection. In general, the method need to fulfill a nontrivial bijection between $\mathcal{E}_{n, k}$ and $\mathcal{S}_{n}$ for every $k$. In our method, the generating function has already guaranteed the property of uniform partition. So we need $\mathcal{E}_{n, k} \cong \mathcal{S}_{n}$ for only one $k$ and usually this bijection is very trivial.

To accomplish the mission mentioned above, we shall employ the functional equation involving $G$. In this paper, we only focus on some generating function $G(x)$ together with $A=A_{G}(x)$ satisfying

$$
x=\frac{P(A)}{Q(A)},
$$

where $P$ and $Q$ are polynomials. We simply name (4) the fraction condition for $G$.

For instance, let $C^{(d)}(x)=\sum_{n \geq 0} c_{n}^{(d)} x^{d n}$ be the generating function of the generalized Catalan numbers of order $d .^{\mathrm{a}}$ It is well-known that $C^{(d)}(x)=1+x^{d}\left(C^{(d)}(x)\right)^{d+1}$. Multiplying both sides of this functional equation by $x$ and solving for $x$, we get

$$
x=A_{C^{(d)}}-\left(A_{C^{(d)}}\right)^{d+1} .
$$

As for the generating function $M(x)$ of the Motzkin numbers, it satisfies the functional equation $M=1+x M+x^{2} M^{2}$. By similar calculation, we obtain

$$
x=\frac{A_{M}}{1+A_{M}+A_{M}^{2}} .
$$

\footnotetext{
${ }^{\text {a }}$ The generating function defined as $\sum_{n \geq 0} c_{n}^{(d)} x^{n}$ is not proper here, because the extension rate is supposed to be $d n+1$.
} 
So the both known CF type results satisfy the fraction condition (4).

In the rest of the paper we always assign $z=x y$, and let $A=A_{G}:=x G(x)$ and $\bar{A}=A(z)$ for convenience. When $x=\frac{P(A)}{Q(A)}$ is provided, the function $\mathrm{CF}_{G}$ can be obtained by the following manipulation. Let $P_{x}=P(A)$ and $P_{z}=P(\bar{A})$ for short, and $Q_{x}, Q_{z}$ are defined similarly. Given a polynomial $F(A)$ (whose variable is $A$, while $A=A(x)$ is a formal power series), we define

$$
\hat{F}=\hat{F}(A, \bar{A}):=\frac{F(A)-F(\bar{A})}{A-\bar{A}},
$$

which is again a polynomial with variables $A$ and $\bar{A}$, because $A-\bar{A}$ must be a factor of $F(A)-F(\bar{A})$. Clearly $\hat{F}(A, \bar{A})=\hat{F}(\bar{A}, A)$ by definition. Now we derive that

$$
\begin{aligned}
x-z & =\frac{P_{x}}{Q_{x}}-\frac{P_{z}}{Q_{z}} \\
& =\frac{\left(P_{x} Q_{z}-P_{x} Q_{x}\right)+\left(P_{x} Q_{x}-P_{z} Q_{x}\right)}{Q_{x} Q_{z}} \\
& =\frac{-P_{x} \hat{Q}(A-\bar{A})+\hat{P} Q_{x}(A-\bar{A})}{Q_{x} Q_{z}}
\end{aligned}
$$

Plugging the identity above into the definition (3), we obtain

$$
\mathrm{CF}_{G}(x, y)=\frac{Q_{x} Q_{z}}{\hat{P} Q_{x}-P_{x} \hat{Q}}
$$

The following proposition provides more equivalent formulas.

Proposition 2.2 Suppose $G(x)$ be a formal power series. Adopt the definition of $A$, $P_{x}, P_{z}, \hat{P}$ and $\hat{Q}$ where $z=x y$ as before. If $x=\frac{P(A)}{Q(A)}$, then the generating function of Chung-Feller extension with respect to $G(x)$ can be represented as

$$
\begin{aligned}
\mathrm{CF}_{G}(x, y) & =\frac{Q_{x} Q_{z}}{\hat{P} Q_{x}-P_{x} \hat{Q}} \\
& =\frac{Q_{z}}{\hat{P}-x \hat{Q}} \\
& =\frac{\frac{P_{x}}{x} \frac{P_{z}}{z}}{\hat{P} Q_{x}-P_{x} \hat{Q}}
\end{aligned}
$$

One of (8)-(10) reveals a possible sup-structure class to be a CF extension. For practice, let us re-prove Theorem 1.1 as follows.

We need some new notation here. Let $v$ be a point on path $P$ or an integral $x$ coordinate in the span of $P$. We define $P_{v}^{L}$ and $P_{v}^{R}$ respectively to be the left and the right subpaths of $P$ cut by $v$. Also $P_{[u, v]}:=P_{u}^{R} \cap P_{v}^{L}$ is the subpath of $P$ in between $u$ and $v$. 
A new proof for Theorem 1.1 We only prove the the unform-partition property and then Eq. (1) follows immediately. By (5), we have $P(A)=A-A^{d+1}$ and $Q(A)=1$. Then $\hat{P}(A, \bar{A})=1-\sum_{i=0}^{d} A^{i} \bar{A}^{d-i}$ and $\hat{Q}(A, \bar{A})=0$. By (9), we obtain

$$
\mathrm{CF}_{C^{(d)}}(x, y)=\frac{1}{1-\sum_{i=0}^{d} A^{i} \bar{A}^{d-i}}=\sum_{m \geq 0}\left(\sum_{i=0}^{d} A^{i} \bar{A}^{d-i}\right)^{m} .
$$

We explain that $\mathrm{CF}_{C^{(d)}}(x, y)$ is exactly the generating function of $\mathcal{C}_{n, k}^{(d)}$ for $n \geq 0$ and $0 \leq k \leq d n$ as follows. Given any $P \in \mathcal{L}_{(d+1) \mathbb{N}, S_{\mathcal{C}(d)}}$, suppose $P$ has $m$ steps $D_{d}$ intersecting the $x$-axis. In particular, $m=0$ if and only if $P$ is the path of length 0 . For each of these $D_{d}$, let $u$ and $v$ be its left and right end points. Let us mark the first intersection point between the subpath $P_{v}^{R}$ and the $x$-axis. No doubt that the last marked point is exactly the right end point of $P$, and then these $m$ marked points cut $P$ into $m$ subpaths. Each of these subpaths contained a unique $D_{d}$ intersecting the $x$-axis. According to (11), each of these $m$ subpaths should be represented by a term $x^{a} y^{b}$ (with coefficient 1 ) in $\sum_{i=0}^{d} A^{i} \bar{A}^{d-i}$. In other words, $\sum_{i=0}^{d} A^{i} \bar{A}^{d-i}$ stands for a GF of all possibilities for this single subpath. We need more detail to identify each other.

Let $Q$ be one of these $m$ subpaths. Here we not only consider this single subpath $Q$ but also all possibilities for $Q$. There is a unique $D_{d}$ intersecting the $x$-axis on $Q$, and suppose that the $y$-coordinates of the two end points $u, v$ of this $D_{d}$ are $i$ and $i-d$ respectively $(0 \leq i \leq d)$. Let us consider $Q_{u}^{L}$ and $Q_{v}^{R}$. Note that isomorphically $Q_{u}^{L}$ is from $(0,0)$ to $(*, i)$ and $Q_{v}^{R}$ is from $(0, i-d)$ to $(*, 0)$, and they never touch the $x$-axis except their end points, i.e., they are exactly the two parts of $Q$ over and below the $x$-axis. A routine technique for lattice paths is to cut $Q_{u}^{L}$ into $i$ pieces according the left end point of the last step $U$ intersecting the line $y=k$ for $k=1, \ldots, i-1$. Each of these $i$ pieces is a step $U$ followed by a Catalan path; so $A=x G$ generates a single piece. Therefore, the all possibilities for $Q_{u}^{L}$ can be represented by $A^{i}$. Similarly, the all possibilities for $Q_{w}^{R}$ can be represented by $A^{d-i}$.

However, it is $\bar{A}^{d-i}$ rather than $A^{d-i}$ appearing in $\mathrm{CF}_{C^{(d)}}(x, y)$; so we realize that the exponent of $y$ counts the number of the steps $U$ on $Q_{w}^{R}$, which are exactly the flaw steps $U$ on $Q$. Since $Q$ is combined by three parts, $Q_{u}^{L}, D_{d}$ and $Q_{v}^{R}$, and $D_{d}$ responses for neither $x$ 's nor $y$ 's powers, the bivariate GF of $Q$ is then $\sum_{i=0}^{d} A^{i} \bar{A}^{d-i}$.

Combining $m$ subpaths with each similar to $Q$ and running $m$ from 0 to $\infty$, we obtain $\sum_{m \geq 0}\left(\sum_{i=0}^{d} A^{i} \bar{A}^{d-i}\right)^{m}$ as the bivariate generating function of $\mathcal{L}_{(d+1) \mathbb{N}, S_{\mathcal{C}^{(d)}}}$, where the power indices of $x$ and $y$ represent the numbers of all steps $U$ and the flaw ones respectively. The proof is now complete.

\subsection{Chung-Feller property of the Motzkin paths}

The set $\mathcal{M}_{n}$ of the well-known Motzkin $n$-paths is exactly $\mathcal{L}_{n, S_{\mathcal{M}}}^{+}$with step set $S_{\mathcal{M}}=\{U=$ $(1,1), D=(1,-1), L=(1,0)\}$. The cardinality $m_{n}$ of $\mathcal{M}_{n}$ is called the $n$th Motzkin number. Here we deal with the sup-structure set $\mathcal{L}_{(n+1,1), S_{\mathcal{M}}}$. Let $\mathcal{H}_{n, k} \subseteq \mathcal{L}_{(n+1,1), S_{\mathcal{M}}}$ 
$(0 \leq k \leq n)$ consist of those paths whose rightmost minima occurring at $x=k$. Clearly, $\mathcal{L}_{(n+1,1), S_{\mathcal{M}}}=\biguplus_{k=0}^{n} \mathcal{H}_{n, k}$ and one can easily map $\mathcal{H}_{n, 0}\left(\mathcal{H}_{n, n}\right)$ to $\mathcal{M}_{n}$ isomorphically by deleting the first (last) step of each path. Not only these two particular cases, but also

$$
\left|\mathcal{H}_{n, k}\right|=\left|\mathcal{M}_{n}\right|=m_{n}
$$

for all $k$, i.e., $\mathcal{L}_{(n+1,1), S_{\mathcal{M}}}$ is partitioned uniformly into $\left\{\mathcal{H}_{n, k}\right\}_{k=0}^{n}$. As a variation of ChungFeller theorem, this problem was first noted by Shapiro [26], and the extension $\mathcal{L}_{(n+1,1), S_{\mathcal{M}}}$ and blocks $\left\{\mathcal{H}_{n, k}\right\}_{k=0}^{n}$ were suggested by an anonymous referee of his paper. A proof using bijection was given by Eu et al. [11]. Here we provide a new proof using generating functions.

By (6), we have $P(A)=A$ and $Q(A)=1+A+A^{2}$, and also $\hat{P}=1$ and $\hat{Q}=1+A+\bar{A}$. Plugging these into (10), we obtain

$$
\mathrm{CF}_{M}(x, y)=\frac{\frac{A}{x} \frac{\bar{A}}{z}}{1-A \bar{A}}=\frac{A}{x} \frac{\bar{A}}{z} \sum_{m \geq 0}(A \bar{A})^{m}=M(z)\left(\sum_{m \geq 0} \bar{A}^{m} A^{m}\right) M(x) .
$$

We analyze the pattern of $M(z)\left(\sum_{m \geq 0} \bar{A}^{m} A^{m}\right) M(x)$ to offer a new proof for the following CF property of the Motzkin paths.

Theorem $2.3([\mathbf{1 1}, \mathbf{2 6}])$ Let $\mathcal{H}_{n, k} \subseteq \mathcal{L}_{(n+1,1), S_{\mathcal{M}}}$ consist of those paths whose rightmost minima occurring at $x=k$. The structure set $\mathcal{L}_{(n+1,1), S_{\mathcal{M}}}$ is partitioned uniformly into $\left\{\mathcal{H}_{n, k}\right\}_{k=0}^{n}$. Therefore, $\mathcal{L}_{(n+1,1), S_{\mathcal{M}}}$ is a CF extension of the Motzkin n-paths.

Proof. Let $\mathcal{L}_{(\mathbb{N}+1,1), S_{\mathcal{M}}}=\biguplus_{n \geq 0} \mathcal{L}_{(n+1,1), S_{\mathcal{M}}}$ and define the bivariate generating function of $\mathcal{L}_{(\mathbb{N}+1,1), S_{\mathcal{M}}}$ by

$$
\sum_{P \in \mathcal{L}_{(\mathbb{N}+1,1), S_{\mathcal{M}}}} x^{\ell(P)-1} y^{\rho(P)},
$$

where $\ell(P)$ is the length of $P$ and $\rho(P)$ is the $x$-coordinate of the rightmost minimum. Once we analyze that the above generating function equals $M(z)\left(\sum_{m \geq 0} \bar{A}^{m} A^{m}\right) M(x)$, we conclude that $\mathcal{L}_{(n+1,1)}$ is a $\mathrm{CF}$ extension of $\mathcal{M}_{n}$ and it can be partitioned uniformly into $\left\{\mathcal{H}_{n, k}\right\}_{k=0}^{n}$.

For any $P \in \mathcal{L}_{(\mathbb{N}+1,1), S_{\mathcal{M}}}$, let $u$ be its rightmost minimum point and let $\bar{U}=[u, v]$ denote the rise step following $u$ immediately. Suppose that the $y$-coordinate of $u$ is $-m$. Notice that $m$ can be any natural number among all paths $P \in \mathcal{L}_{(\mathbb{N}+1,1), S_{\mathcal{M}}}$. On the subpath $P_{u}^{L}$, let us find the first fall step $D$ dropping from $y=-i$ to $y=-i-1$ for $i=0, \ldots, m-1$, and mark the left end points of this $D$ by $u_{i}$. The subscript of $u_{i}$ also indicates the absolute $y$-coordinate of this points. These $m$ points $u_{i}$ partition $P_{u}^{L}$ into $m+1$ subpaths such that the first subpath is a Motzkin path (probably of length 0 ), and each of the rest subpaths begins with a fall step $D$ followed by a Motzkin path over the line $y=-i-1$. Thus, $P_{u}^{L}$ is represented by $M(z) \bar{A}^{m}$. Notice that the exponent of $y$ in $M(z) \bar{A}^{m}$ is exactly the $x$-coordinate of $u$.

On $P_{v}^{R}$ (not $P_{u}^{R}$ ), let us find the last rise step $U$ rising from $y=-j$ to $y=-j+1$ for $j=0, \ldots, m-1$, and mark the right end point of this $U$ by $v_{j}$. Again, these $m$ points 
$v_{j}$ partition $P_{v}^{R}$ into $m+1$ subpaths such that the last one is a Motzkin path (probably of length 0 ), and each of the rest subpaths is a Motzkin path followed by a rise step $U$. Thus, $P_{v}^{R}$ is represented by $A^{m} M(x)$.

According to the interpretation above, the step $\bar{U}=[u, v]$ appears in neither $P_{u}^{L}$ (corresponding to $M(z) \bar{A}^{m}$ ) nor $P_{v}^{R}$ (corresponding to $A^{m} M(x)$ ). However, this $\bar{U}$ is unique in every $P \in \mathcal{L}_{(\mathbb{N}+1,1), S_{\mathcal{M}}}$; so we simply ignore its count as the exponent of $x$ or $y$. This is why the exponent of $x$ in $(13)$ is $\ell(P)-1$. The whole proof is complete new.

A different interpretation of $\mathrm{CF}_{M}(x, y)$. Follows the discussion in the last proof. Let us connect $P_{u}^{L}$ and $P_{v}^{R}$ by contracting $u$ and $v$ into one point. Let $P^{\prime}$ denote this new lattice path and $w$ the new point obtained by contracting $u$ and $v$. Clearly, $P^{\prime} \in \mathcal{L}_{\mathbb{N}, S_{\mathcal{M}}}$ and $w$ is one of the minimum points of $P^{\prime}$, not necessarily the rightmost one. The combination of $P^{\prime}$ and $w$ yields a new interpretation of $\mathrm{CF}_{M}(x, y)$ by defining

$$
\left(\mathcal{L}_{\mathbb{N}, S_{\mathcal{M}}}, \mathcal{W}\right)=\left\{\left(P^{\prime}, w\right) \mid P^{\prime} \in \mathcal{L}_{\mathbb{N}, S_{\mathcal{M}}} \text { and } w \text { is one of the minimum points of } P^{\prime}\right\} .
$$

The bivariate GF of $\left(\mathcal{L}_{\mathbb{N}, S_{\mathcal{M}}}, \mathcal{W}\right)$ shall be defined as $\sum_{\left(P^{\prime}, w\right) \in\left(\mathcal{L}_{\mathbb{N}, S}, \mathcal{W}\right)} x^{\ell\left(P^{\prime}\right)} y^{w_{x}}$, where $w_{x}$ is the $x$-coordinate of $w$. This generating function equals the one in (13), and then equals $\mathrm{CF}_{M}(x, y)$.

Corollary 2.4 The structure set $\left(\mathcal{L}_{n, S_{\mathcal{M}}}, \mathcal{W}\right)$ is a $C F$ extension of $\mathcal{M}_{n}$.

The advantage of the this corollary is that $\mathcal{L}_{n, S_{\mathcal{M}}}$ is the set of free Motzkin $n$-paths.

\section{Chung-Feller property for a multivariate GF}

Now we consider some sub-structure sets that admits multivariate generating functions.

Definition 3.1 Let $\mathbf{G}\left(x_{1}, \ldots, x_{k}\right)=\sum_{n_{1}, \ldots, n_{k}>0} a_{n_{1}, \ldots, n_{k}} x_{1}^{n_{1}} \cdots x_{k}{ }^{n_{k}}$ be the multivariate generating function of a sequence $\left\{a_{n_{1}, \ldots, n_{k}}\right\}_{n_{1}, \ldots, n_{k} \geq 0}$ and $A_{\mathbf{G}}=x_{1} \mathbf{G}\left(x_{1}, \ldots, x_{k}\right)$. The function of Chung-Feller extension with respect to $\mathbf{G}$ and $x_{1}$ is denoted and defined as

$$
\mathrm{CF}_{\mathbf{G}, x_{1}}\left(x_{1}, \ldots, x_{k}, y\right)=\left.\frac{A_{\mathbf{G}}\left(x_{1}, x_{2}, \ldots, x_{k}\right)-A_{\mathbf{G}}\left(z, x_{2}, \ldots, x_{k}\right)}{x_{1}-z}\right|_{z=x_{1} y} .
$$

This definition is due to that we are looking for a sup-structure class with the generating function

$$
\sum_{n_{1}, \ldots, n_{k} \geq 0} a_{n_{1}, \ldots, n_{k}} x_{1}^{n_{1}} \cdots x_{k}^{n_{k}}\left(1+y+\cdots+y^{n_{1}}\right) .
$$

According to (15) as well as (14), a sup-structure set is partitioned into $n_{1}+1$ blocks, corresponding to $y^{i}$ for $i=0, \ldots, n_{1}$, of uniform size $a_{n_{1}, \ldots, n_{k}}$ for every fixed $k$-tuple $\left(n_{1}, \ldots, n_{k}\right)$. According (15), the extension rate is independent on $n_{2}, \ldots, n_{k}$. It is easy to check that Proposition 2.2 still holds for $\mathrm{CF}_{\mathbf{G}, x_{1}}$ by replacing $A$ with $A_{\mathbf{G}}$ and defining the corresponding $\bar{A}_{\mathbf{G}}, P_{x}, P_{z}, \hat{P}$ and $\hat{Q}$ similarly. 
Let $\mathcal{S}_{\mathbb{N}^{k}}=\biguplus_{n_{1}, \ldots, n_{k} \geq 0} \mathcal{S}_{n_{1}, \ldots, n_{k}}$ be a structure class such that $\left|\mathcal{S}_{n_{1}, \ldots, n_{k}}\right|=a_{n_{1}, \ldots, n_{k}}$. If there exists a sup-structure class admitting $\mathrm{CF}_{\mathbf{G}, x_{1}}$ as its generating function, we shall call this sup-structure class a Chung-Feller extension of $\mathcal{S}_{\mathbb{N}^{k}}$ along the first index or associating the quantity counted by the first index. We say "first index" because the extension rate is $n_{1}+1$ according to the first sub-index of $\mathcal{S}_{n_{1}, \ldots, n_{k}}$.

\subsection{Generalized Motzkin paths}

We consider step set $S_{\mathcal{M}^{(d)}}:=\left\{U=(1,1), D_{d}=(1,-d), L=(1,0)\right\}$ to construct generalized Motzkin paths of order d. ${ }^{\mathrm{b}}$ Obviously, this generalization is motivated by the generalized Catalan numbers of order $d$. Given $n, m \in \mathbb{N}$ with $0 \leq m \leq n$, let $\mathcal{L}_{n, m, S_{\mathcal{M}^{(d)}}}$ consist of free generalized Motzkin $n$-paths with exactly $m$ steps $L$, and $\mathcal{M}_{n, m}^{(d)}=: \mathcal{L}_{n, m, S_{\mathcal{M}^{(d)}}^{+}}$. Also let $\mathcal{M}_{\mathbb{N}^{2}}^{(d)}=\biguplus_{n, m \geq 0} \mathcal{M}_{n, m}^{(d)}$. Define a multivariate generating function for the class $\mathcal{M}_{\mathbb{N}}^{(d)}$ as

$$
\mathbf{M}(x, s, t)=\sum_{P \in \mathcal{M}_{\mathbb{N}^{2}}^{(d)}} x^{\ell(P)} s^{U(P)} t^{L(P)},
$$

where $U(P)$ and $L(P)$ are respectively the numbers of rise steps $U$ and level steps $L$ on $P$. To record the number of fall steps $D_{d}$ is unnecessary, because it equals $\ell(P)-U(P)-L(P)$. We should use only one of $U(P)$ and $L(P)$ because $U(P)=\frac{d}{d+1}(\ell(P)-L(P))$; however, we keep both of them because we can trace $s$ as step $U$ 's footprint in order to distinguish $U$ from $D_{d}$ in the following discussion, and we use $t^{L(P)}$ to deal with Catalan paths and generalized $k$-color Motzkin paths.

Let $A=A_{\mathbf{M}}:=x \mathbf{M}(x, s, t)$ and $\bar{A}=A_{\mathbf{M}}(z, s, t)$. It is easy to derive the functional equation $\mathbf{M}=1+t x \mathbf{M}+s^{d} x^{d+1} \mathbf{M}^{d+1}$ by considering three types of paths: of length 0 , with first step $L$, and with first step $U$. Then we obtain the fraction condition as

$$
x=\frac{A}{1+t A+s^{d} A^{d+1}} .
$$

With $P(A)=A$ and $Q(A)=1+t A+s^{d} A^{d+1}$, we get $\hat{P}=1$ and $\hat{Q}=t+s^{d} \sum_{k=0}^{d} A^{k} \bar{A}^{d-k}$. By (10), we derive that

$$
\begin{aligned}
\mathrm{CF}_{\mathbf{M}, x}(x, s, t, y) & =\frac{\frac{A}{x} \frac{\bar{A}}{z}}{1-s^{d} \sum_{i=1}^{d} A^{i} \bar{A}^{d-i+1}} \\
& =\mathbf{M}(x, s, t) \mathbf{M}(z, s, t) \sum_{m \geq 0}\left(\bar{A} \sum_{i=1}^{d}(s A)^{i}(s \bar{A})^{d-i}\right)^{m} .
\end{aligned}
$$

It is easier to interpret $\mathrm{CF}_{\mathbf{M}, x}$ by a similar form given in Corollary 2.4 rather than Theorem 2.3. Let us define a sup-structure class as

$$
\left(\mathcal{L}_{\mathbb{N}^{2}, S_{\mathcal{M}}(d)}, \mathcal{W}\right):=\left\{(P, w) \mid P \in \mathcal{L}_{\mathbb{N}^{2}, S_{\mathcal{M}}(d)} \text { and } w \text { is a minimum point of } P\right\} .
$$

\footnotetext{
${ }^{\mathrm{b}}$ There is another kind of generalized Motzkin paths defined by the step set $\{U=(1,1), D=$ $(1,-1), L=(h, 0)\}$ (see [2]).
} 
The point $w$ is called the marked minimum. A new Chung-Feller type result is given as follows:

Theorem 3.2 The structure set $\left(\mathcal{L}_{n, m, S_{\mathcal{M}^{(d)}}}, \mathcal{W}\right)$ can be uniformly partitioned into $n+1$ blocks according to the $x$-coordinates of marked minima. Therefore, $\left(\mathcal{L}_{n, m, S_{\mathcal{M}}^{(d)}}, \mathcal{W}\right)$ is a Chung-Feller extension of $\mathcal{M}_{n, m}^{(d)}$ associating the length with extension rate $n+1$.

Proof. Let us denote $\left.\mathcal{J}=\left(\mathcal{L}_{\mathbb{N}^{2}, S} \mathcal{M}^{(d)}\right), \mathcal{W}\right)$ for short and define a multivariate GF of $\mathcal{J}$ by

$$
\mathrm{G}_{\mathcal{J}}(x, s, t, y):=\sum_{(P, w) \in \mathcal{J}} x^{\ell(P)} s^{U(P)} t^{L(P)} y^{w_{x}},
$$

where $w_{x}$ is the $x$-coordinate of $w$ (similarly, for $w_{y}$ ). To prove this theorem, we shall verify that $\mathrm{CF}_{\mathrm{M}, x}=\mathrm{G}_{\mathcal{J}}$.

The following proof is modified from the one for Theorem 2.3. We simply decompose any $(P, w) \in \mathcal{J}$ piece by piece as follows. On the horizontal line $y=w_{y}$, there are isomorphically two pieces of generalized Motzkin paths $P_{[u, w]}$ and $P_{[w, v]}$, where $u$ and $v$ are respectively the leftmost and the rightmost minima on $P$. Clearly, the kinds (possibilities) of $\left(P_{[u, w]}, P_{[w, v]}\right)$ claim their $\mathrm{GF}$ as $\mathbf{M}(z, s, t)$ and $\mathbf{M}(x, s, t)$.

Connect $P_{u}^{L}$ and $P_{v}^{R}$ by contracting $u$ and $v$ into one point. Let $P^{\prime}$ denote this new path and $w^{\prime}$ the point of contraction, which is the unique minimum on $P^{\prime}$. Also let $D_{d}=\left[w^{\prime \prime}, w^{\prime}\right]$ be the fall step reaching $w^{\prime}$, and $u^{\prime}$ be the leftmost minimum on $P_{w^{\prime \prime}}^{L}$. In addition, let $v^{\prime}$ be the last point that $P^{\prime}$ intersects the line $y=u_{y}^{\prime}$. Suppose $i=u_{y}^{\prime}-w_{y}^{\prime}$. Clearly, $1 \leq i \leq d$. For a fixed $i$, It is a routine check that the kinds of $P^{\prime}{ }_{\left[u^{\prime}, v^{\prime}\right]}$ claim their GF as $\bar{A}(s A)^{i}(s \bar{A})^{d-i}$. Precisely, $(s \bar{A})^{d-i}$ represents $P^{\prime}{ }_{\left[u^{\prime}, b\right]}$, where $R=[a, b]$ is the last rise step on $P_{\left[u^{\prime}, w^{\prime \prime}\right]}^{\prime}$ such that $b_{y}=w_{y}^{\prime \prime}$. Note that $b=w^{\prime \prime}$ is possible. Moreover, $\bar{A}$ represents $P^{\prime}{ }_{\left[b, w^{\prime}\right]}$ and $(s A)^{i}$ represents $P_{\left[w^{\prime}, v^{\prime}\right]}^{\prime}$. Here we find that $s$ is a clear footprint of step $U$. Therefore, the kinds of $P^{\prime}{ }_{\left[u^{\prime}, v^{\prime}\right]}$ for all $1 \leq i \leq d$ claim their GF as $\bar{A} \sum_{i=1}^{d}(s A)^{i}(s \bar{A})^{d-i}$.

Now connect $P_{u^{\prime}}^{L}$ and $P_{v^{\prime}}^{R}$ by contracting $u^{\prime}$ and $v^{\prime}$ into one point, and do the same work as the last paragraph until we get a single point. It is now clear that why there is a exponent $m$ in Eq. (18). The proof of $\mathrm{CF}_{\mathbf{M}, x}=\mathrm{G}_{\mathcal{J}}$ is now complete.

Let $\mathcal{L}_{(n+1,1), m, S_{\mathcal{M}^{(d)}}}$ consist of free generalized Motzkin paths ending at $(n+1,1)$ with exactly $m$ step $L$. We immediately get the next result by the above theorem.

Corollary 3.3 The structure set $\mathcal{L}_{(n+1,1), m, S_{\mathcal{M}^{(d)}}}$ can be uniformly partitioned into $n+1$ blocks according to the $x$-coordinates of rightmost minima. Therefore, $\mathcal{L}_{(n+1,1), m, S_{\mathcal{M}^{(d)}}}$ is a Chung-Feller extension of $\mathcal{M}_{n, m}^{(d)}$ with extension rate $n+1$.

From the view of the above two results, Theorem 2.3 and Corollary 2.4 now become the special case as $d=1$ and also all $m(0 \leq m \leq n)$ are considered at a time.

Note that $\mathbf{M}(x, s, 0)=C^{(d)}\left(x^{d+1} s^{d}\right)$, because plugging $t=0$ forces us considering only those paths without step $L$, which are the Catalan paths of order $d$. Here, we derive a various kind of extension for $\mathcal{C}_{n}^{(d)}$. It is various compared with the previous know $\mathrm{CF}$-properties for the extension rates are different. 
Corollary 3.4 The structure sets $\mathcal{L}_{((d+1) n+1,1), S_{\mathcal{C}^{(d)}}}$ and $\left(\mathcal{L}_{(d+1) n, S_{\mathcal{C}^{(d)}}}, \mathcal{W}\right)$ are both ChungFeller extensions of $\mathcal{C}_{n}^{(d)}$ associating the length of the path with extension rate $d n+n+1$.

By this CF property, one easily derives a various formula for the generalized Catalan number:

$$
c_{n}^{(d)}=\frac{1}{d n+n+1}\left(\begin{array}{c}
d n+n+1 \\
n
\end{array}\right) .
$$

Particularly when $d=1$, we get

$$
c_{n}=\left|\mathcal{D}_{n}\right|=\frac{1}{2 n+1}\left(\begin{array}{c}
2 n+1 \\
n
\end{array}\right) .
$$

A bijective proof of this identity was given in [31].

\subsection{Generalized $k$-color Motzkin paths}

Let $k$ be a fixed positive integer. By assigning one of $k$ different colors for each step $L$ on a normal Motzkin path, one defines a $k$-colored Motzkin path (see $[10,25]$ ). By the same way, we can define generalized $k$-color Motzkin paths of order $d$, and denote the corresponding set by a $\mathcal{C} \mathcal{M}_{n}^{(k, d)}$ and $\mathcal{C} \mathcal{M}_{n, m}^{(k, d)}$. In particular, we have $\mathcal{C} \mathcal{M}_{n}^{(1, d)}=\mathcal{M}_{n}^{(d)}$ and $\mathcal{C} \mathcal{M}_{n, m}^{(1, d)}=\mathcal{M}_{n, m}^{(d)}$.

Assigning $k$ colors is equivalent to enlarging both $\mathcal{M}_{n, m}^{(d)}$ and $\mathcal{L}_{(n+1,1), m, S_{\mathcal{M}^{(d)}}}$ by $k^{m}$ times. Therefore, $\mathcal{C} \mathcal{M}_{n, m}^{(k, d)}$ has $k$-color $\mathcal{L}_{(n+1,1), m, S_{\mathcal{M}^{(d)}}}$ as its $\mathrm{CF}$ extension with rate $n+1$ by Corollary 2.4 .

Actually, we can derive more than that by considering the generating function

$$
\begin{aligned}
\mathbf{C M}\left(x, s, L_{1}, \ldots, L_{k}\right) & :=\sum_{\ell, r, c_{1}, \ldots, c_{k} \geq 0} m_{\ell, r, c_{1}, \ldots, c_{k}} x^{\ell} s^{r} L_{1}^{c_{1}} \cdots L_{k}^{c_{k}} \\
& =\mathbf{M}\left(x, s, L_{1}+\cdots+L_{k}\right),
\end{aligned}
$$

where $m_{\ell, r, c_{1}, \ldots, c_{k}}$ is the number of the generalized $k$-color Motzkin paths of order $d$ whose length are $\ell$, and who have $r$ steps $U$ and $c_{1}, \ldots, c_{k}$ steps $L$ colored respectively by $1, \ldots, k$. In (20), $\mathbf{M}$ is defined as in (16) and replacing $t$ by $L_{1}+\cdots+L_{k}$ is a trivial trick. The generating function of Chung-Feller extension along the first index with respect to CM is

$$
\begin{aligned}
\mathrm{CF}_{\mathbf{C M}}\left(x, s, L_{1}, \ldots, L_{k}, y\right) & :=\sum_{\ell, r, c_{1}, \ldots, c_{k}} m_{\ell, r, c_{1}, \ldots, c_{k}} x^{\ell} s^{r} L_{1}^{c_{1}} \cdots L_{k}^{c_{k}}\left(1+y+\cdots+y^{\ell}\right) \\
& =\mathrm{CF}_{\mathbf{M}, x}\left(x, s, L_{1}+\cdots+L_{k}, y\right),
\end{aligned}
$$

Note that the first identity is by definition and the second one follows (20).

Let $\mathcal{C M}_{n, c_{1}, \ldots, c_{k}}^{(k, d)} \subseteq \mathcal{C M}_{n}^{(k, d)}$ consist of all $n$-paths that have $c_{1}, \ldots, c_{k}$ steps $L$ colored

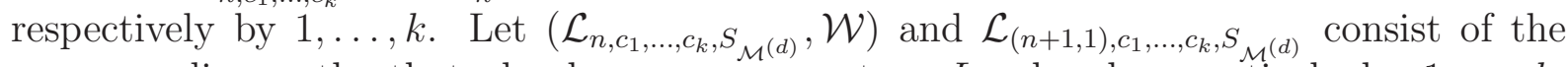
corresponding paths that also have $c_{1}, \ldots, c_{k}$ steps $L$ colored respectively by $1, \ldots, k$. By (21), the interpretation of $\mathrm{CF}_{\mathbf{C M}}$ can be easily followed from Theorem 3.2 and Corollary 3.3. Therefore, we conclude a general result: 
Theorem 3.5 The structure sets $\left(\mathcal{L}_{n, c_{1}, \ldots, c_{k}, S_{\mathcal{M}^{(d)}}}, \mathcal{W}\right)$ and $\mathcal{L}_{(n+1,1), c_{1}, \ldots, c_{k}, S_{\mathcal{M}^{(d)}}}$ are both Chung-Feller extensions of $\mathcal{C M}_{n, c_{1}, \ldots, c_{k}}^{(k, d)}$ with extension rate $n+1$.

Remark We can also extend $\mathbf{M}(x, s, t)$ along its second index. By the functional identity $\mathbf{M}=1+t x \mathbf{M}+s^{d} x^{d+1} \mathbf{M}^{d+1}$ given in Subsection 3.1, we get

$$
s=(1-t x) A-x^{d+1} A^{d+1},
$$

where $A:=s \mathbf{M}$. In particular, provided $t=0$ (which means no $L$ steps), $x=1$ and then $s$ replaced by $x$, this fraction condition will agree with (5). Thus, we expect to re-derive the classical Chung-Feller theorem in this special case. Indeed, (22) can support another version of CF-property for the generalized Motzkin paths of order $d$. We leave this as an exercise or the reader may refer to [19].

\section{The Schröder paths}

We explore another two examples of the Chung-Feller property in the rest of the paper. Let $\mathcal{S C}_{n}=\mathcal{L}_{2 n, S_{\mathcal{S C}}}^{+}$with $S_{\mathcal{S C}}=\left\{U=(1,1), D=(1,-1), L_{2}=(2,0)\right\}$. These lattice paths are called the (large) Schröder $n$-paths and the cardinality $\left|\mathcal{S C}_{n}\right|$ is the $n$th Schröder number (see $[24,30])$. Note that $n$ means the semi-length here. Let

$$
\mathbf{S C}(x, u, w)=\sum_{P \in \mathcal{S} \mathcal{C}_{\mathbb{N}}} x^{\ell(P) / 2} u^{U(P)} w^{L_{2}(P)}
$$

be the generating function of the Schröder paths. The benefit by adopting exponent $U(P)$ is mentioned at the end of the last section. It is easy to derive that $\mathbf{S C}=1+x w \mathbf{S C}+$ $x u \mathbf{S C}^{2}$, where $x w$ represents a level step $L_{2}$ and $x u$ represents a $U$ together with a $D$. So we have

$$
x=\frac{A-u A^{2}}{1+w A}
$$

where $A=A(x, u, w):=x \mathbf{S C}(x, u, w)$, and then $P=A-u A^{2}, Q=1+w A, \hat{P}=$ $1-u A-u \bar{A}$, and $\hat{Q}=w$. By (8), we obtain

$$
\begin{aligned}
\mathrm{CF}_{\mathbf{S C}, x}(x, u, w, y) & =\frac{1+w A+w \bar{A}+w^{2} A \bar{A}}{1-u A-u \bar{A}-u w A \bar{A}} \\
& =\left(1+w A+w \bar{A}+w^{2} A \bar{A}\right) \sum_{m \geq 0}(u A+u \bar{A}+u w A \bar{A})^{m}
\end{aligned}
$$

To explore the structure represented by $\mathrm{CF}_{\mathbf{S C}, x}$, we separate the formula above into two parts. One is $\mathrm{CF}^{a}(x, u, w)$ that contains only powers of $A$ and no powers of $\bar{A}$ (so it 
is independent on $y$ ) and the other is $\mathrm{CF}^{b}(x, u, w, y)$. Precisely, we have

$$
\begin{aligned}
\mathrm{CF}^{a}= & (1+w A) \sum_{m \geq 0}(u A)^{m} \\
\mathrm{CF}^{b}= & (1+w A)\left(\sum_{m_{1} \geq 0} u A^{m_{1}}\right)(u \bar{A}+u w A \bar{A}) \sum_{m_{2} \geq 0}(u A+u \bar{A}+u w A \bar{A})^{m_{2}} \\
& \quad+\left(w \bar{A}+w^{2} A \bar{A}\right) \sum_{m \geq 0}(u A+u \bar{A}+u w A \bar{A})^{m} \\
= & \left.\quad \mathrm{CF}^{a} u \bar{A}+\mathrm{CF}^{a} u A w \bar{A}+w \bar{A}+w A w \bar{A}\right] \\
& \quad \times \sum_{m \geq 0}(u A+u \bar{A}+u A w \bar{A})^{m}
\end{aligned}
$$

Because $\mathrm{CF}^{a}(x, u, w)=\mathrm{CF}_{\mathbf{S C}, x}(x, u, w, 0)=\mathbf{S C}(x, u, w)$, we shall explore how $\mathrm{CF}^{a}$ interprets the Schröder paths. As a benefit, this interpretation triggers our argument on $\mathrm{CF}^{b}$.

The formula $(1+w A) \sum_{m \geq 0}(u A)^{m}$ symbolizes the last-prairie decomposition of every path in $\mathcal{S C}_{\mathbb{N}}$, where a prairie means a step $L_{2}$ on the $x$-axis (or the horizon). Clearly,

$$
u A=u x \mathbf{S C}=\text { the GF of the paths in form " } U \text {-a Schröder path- } D . "
$$

and we call a path or subpath in this form a hill. If a path $P \in \mathcal{S C}_{\mathbb{N}}$ has no prairie, then it is a combination of contiguous hills; thus, $P$ is one of $1 \times \sum_{m \geq 0}(u A)^{m}$. If $P$ has a prairie, then it is one of $w A \times \sum_{m \geq 0}(u A)^{m}$, where the $w x$ in $w A=w x \mathbf{S C}$ is what we call the last-prairie on $P$. In other words,

$$
w A=\text { the GF of the paths in form "a Schröder path- } L_{2} . "
$$

The way we interpret $w A$ is the key for the argument on $\mathrm{CF}^{b}$. In addition, a subpath related to $u \bar{A}$ is called a vale, because

$$
u \bar{A}=\text { the GF of the paths in form "D-a weakly flaw Schröder path- } U . "
$$

Here is one more tool we need. In (25), the summation of the trinomial powers is rearranged as follows

$$
\begin{aligned}
G & :=\sum_{m \geq 0}(u A+u \bar{A}+u A w \bar{A})^{m} \\
& =\left(\sum_{m_{1} \geq 0}(u A+u \bar{A})^{m_{1}}\right)(1+u A w \bar{A} G) .
\end{aligned}
$$

We claim that $G$ is the generating function of the structure set denoted and defined as

$$
\mathcal{G}:=\left\{P \in \mathcal{L}_{2 \mathbb{N}, S_{\mathcal{S C}}} \mid \text { there is no prairie before the first hill of } P\right\} .
$$

A easy consequence of this claim is that the exponent of $y$ in $G$ equals the number of prairies and flaw steps $D$ on each $P \in \mathcal{G}$. 
Now let us verify the claim. By definition, $P$ shall begin with a $U$ or a $D$; otherwise $\ell(P)=0$. If $P$ has no prairie, then it is a combination of hills and vales; so $P$ is one of $\sum_{m_{1}>0}(u A+u \bar{A})^{m_{1}} \times 1$ in $(27)$. Suppose $P$ has a prairie and a step $L_{2}$ located in $[v, v+2]$ is the first prairie. By definition, some hills exist on $P_{v}^{L}$. Among these hills, suppose the last one lies over the interval $\left[h_{0}, h_{1}\right]$. Starting at $v$ to the right until the next hill or until the end of $P$ if the next hill does not exist, we locate the last prairie $L_{2}$ in the interval $[w-2, w]$. It is easy to realize that subpath $P_{\left[h_{0}, w\right]}$ is one of $u A w \bar{A}$, and the subpath $P_{h_{0}}^{L}$ is one of $\sum_{m_{1}>0}(u A+u \bar{A})^{m_{1}}$. Since the subpath $P_{w}^{R}$ is again the type that before the first hill there is no prairie, it is one of $G$. Therefore, the whole $P$ is one of $\sum_{m_{1} \geq 0}(u A+u \bar{A})^{m_{1}} \times u A w \bar{A} G$ in (27). Now our claim follows.

We define a new structure set as

$$
\begin{aligned}
\left(\mathcal{L}_{2 \mathbb{N}, S_{\mathcal{S C}}}, \mathcal{V}\right):= & \left\{(P, v) \mid P \in \mathcal{L}_{2 \mathbb{N}, S_{\mathcal{S C}}} \text { and } v \text { is a marked point on } P\right. \text { such that } \\
& \left.P_{v}^{L} \text { is a Schröder path and the succeeding step of } v \text { is not } U\right\} .
\end{aligned}
$$

By definition, $v$ must be an even integer point on the $x$-axis, and either it is the right end of $P$ or its succeeding step is $D$ or $L_{2}$. We claim that the generating function of $\left(\mathcal{L}_{2 \mathbb{N}, S_{\mathcal{S C}}}, \mathcal{V}\right)$ is exactly $\mathrm{CF}_{\mathbf{S C}, x}(x, u, w, y)$, where the exponent of $y$ counts the number of flaw steps $D$ and prairies $L_{2}$ on the subpath $P_{v}^{R}$ (or the semi-length of all weakly flaw steps on $P_{v}^{R}$ ) -we will call these steps the $y$-related steps for short. For independent on $y$, $\mathrm{CF}^{a}$ should be the generating function of those $(P, v) \in\left(\mathcal{L}_{2 \mathbb{N}, S_{\mathcal{S C}}}, \mathcal{V}\right)$ that have $v$ locating at the end of $P$. It is correct. Because $P_{v}^{L}$ is a normal Schröder path and the fact $P=P_{v}^{L}$ verifies the set of this kind of $(P, v)$ isomorphic to $\mathcal{S C}_{\mathbb{N}}$.

Now we are led to verify that the rest of $(P, v) \in\left(\mathcal{L}_{2 \mathbb{N}, S_{\mathcal{S C}}}, \mathcal{V}\right)$, each of which has at least a $y$-related step, yield the generating function $\mathrm{CF}^{b}(x, u, w, y)$. Because a $y$-related step appears, $v$ cannot be the right end of $P$, and then $P_{v}^{R}$ starts with a $D$ or $L_{2}$. The following argument has four cases according to the four terms inside the bracket of (25).

Case I: When no prairie exists before the first hill of $P_{v}^{R}$ or before the end of $P_{v}^{R}$ if no hill exists. In this case, the subpath $P_{v}^{R}$ must start with a $D$, or vale in other words. Suppose this vale located in $[v, q]$. It is clear that $P_{v}^{L}, P_{[v, q]}$ and $P_{q}^{R}$ claim ones of $\mathrm{CF}^{a}$, $u \bar{A}$ and $G$ respectively. Therefore, this case claims the first term in the bracket of (25) multiplied by $G$. Note that the $y$-related steps yields by $u \bar{A}$ and $G$, and then the count starts at $v$.

For the remaining three cases II, III and IV, let $[q-2, q]$ be the location of the last prairie $L_{2}$ before the first hill of $P_{v}^{R}$ or before the end if no hill exists on $P_{v}^{R}$. So $P_{[v, q]}$ is one of $w \bar{A}=z w \mathbf{S C}(z, u, w)$, where the factor $z w$ represents this last prairie. For all four cases, the subpath $P_{q}^{R}$ is one of $G$. In addition, $w \bar{A} G$ represents $P_{v}^{R}$ and is a common factor of the remaining three cases. This common factor is the only source of $y$ 's exponent; so the count of the $y$-related steps starts at $v$ again.

Case II: When the subpath $P_{v}^{L}$ is empty. So nothing need to be multiplied by $w \bar{A} G$. The third term in the bracket of (25) matches this case.

Case III: When the preceding step of $v$ is a $U$. Then $P_{v}^{L}$ claims one of $\mathrm{CF}^{a} u A$.

Case IV: When the preceding step of $v$ is a $L_{2}$. Then $P_{v}^{L}$ claims one of $w A$. 


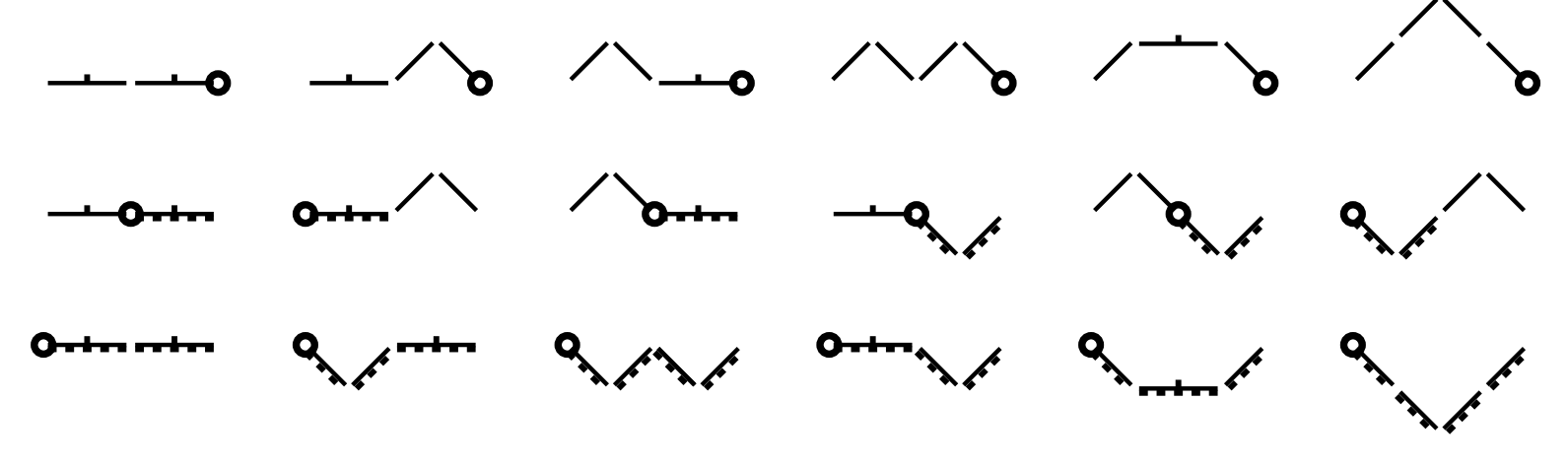

Figure 1: structure set $\left(\mathcal{L}_{4, S_{\mathcal{S C}}}, \mathcal{V}\right)$ and $y$-related steps

The whole proof is now complete. We show $\left(\mathcal{L}_{4, S_{\mathcal{S C}}}, \mathcal{V}\right)$ by Figure 1 and conclude the argument above by the following properties.

Theorem 4.1 (a) The structure $\operatorname{set}\left(\mathcal{L}_{2 n, S_{\mathcal{S C}}}, \mathcal{V}\right)$ is a Chung-Feller extension of the (large) Schröder n-paths. (b) Let $\mathcal{A}_{n, m} \subseteq\left(\mathcal{L}_{2 n, S_{\mathcal{S C}}}, \mathcal{V}\right)$ (respectively $\mathcal{B}_{n, m} \subseteq \mathcal{S C}_{n}$ ) consist of those paths with $m$ steps $L_{2}$. Then we have $\left|\mathcal{A}_{n, m}\right|=(n+1)\left|\mathcal{B}_{n, m}\right| .(c)$ The $k$-colored $\left(\mathcal{L}_{2 n, S_{\mathcal{S C}}}, \mathcal{V}\right)$ (or a weighted one) is a Chung-Feller extension of the $k$-colored (weighted) $\mathcal{S C}_{n}$. (d) Given $\mathbf{m}=\left(m_{1}, \ldots, m_{k}\right)$ with each $m_{i} \in \mathbb{N}$. Let $\mathcal{A}_{n, \mathbf{m}} \subseteq\left(\mathcal{L}_{2 n, S_{\mathcal{S C}}}, \mathcal{V}\right)$ (respectively $\left.\mathcal{B}_{n, \mathbf{m}} \subseteq \mathcal{S C}_{n}\right)$ consist of the paths that have $m_{i}$ steps $L_{2}$ colored by $i$. Then we have $\left|\mathcal{A}_{n, \mathbf{m}}\right|=(n+$ 1) $\left|\mathcal{B}_{n, \mathbf{m}}\right|$.

In part (b), " $k$-colored" means that $L_{2}$ has $k$ different colors, and "weighted" means the same idea of the weight function $\Omega_{w}$ mentioned in the last section.

Given $P \in \mathcal{L}_{2 \mathbb{N}, S_{\mathcal{S C}}}$, a weight function of $P$ is defined by

$$
\Psi(P):=1+\text { the number of prairies before the first flaw step of } P \text {. }
$$

The $\Psi$-weighted $\mathcal{L}_{2 \mathbb{N}, S_{\mathcal{S C}}}$ is an alias of $\left(\mathcal{L}_{2 \mathbb{N}, S_{\mathcal{S C}}}, \mathcal{V}\right)$. The idea of weighting is also demonstrated in Figure 1 where a same path appears as many times as its weight.

Corollary 4.2 The total weight in the $\Psi$-weighted $\mathcal{L}_{2 n, S_{\mathcal{S C}}}$ is $n+1$ times the $n$th Schröder number $\left|\mathcal{S C}_{n}\right|$.

\section{The little Schröder paths}

In this section, we deal with the little Schröder paths that are paths in $\mathcal{S C}_{\mathbb{N}}$ containing no prairie, i.e., no step $L_{2}$ lies on the $x$-axis. We use $\mathcal{L} \mathcal{S}_{n}$ to denote the set of the little Schröder paths of semi-length $n$ and let $\mathbf{L S}(x, u, w)=\sum_{P \in \mathcal{L} \mathcal{S}_{\mathbb{N}}} x^{\ell(P) / 2} u^{U(P)} w^{L_{2}(P)}$ be the generating function. It is easy to derive that $\mathbf{L S}=1+x u \mathbf{S C} \times \mathbf{L S}$, and then

$$
\mathbf{L S}=\frac{1}{1-u A}
$$


where $A=A_{\mathbf{S C}}=x \mathbf{S C}$ not $x \mathbf{L S}$. The approach here is different from all previous $\mathrm{CF}$ extensions in this paper. We have $x \mathbf{L S}=\frac{A}{1+w A}$ by multiplying (29) by $x=\frac{A-u A^{2}}{1+w A}$ (see (23)). By Definition 2.1, we get

$$
\begin{aligned}
\mathrm{CF}_{\mathbf{L S}}(x, u, w, y) & =\frac{x \mathbf{L S}(x)-z \mathbf{L S}(z)}{x-z} \\
& =\left(\frac{A}{1+w A}-\frac{\bar{A}}{1+w \bar{A}}\right) /\left(\frac{A-u A^{2}}{1+w A}-\frac{\bar{A}-u \bar{A}^{2}}{1+w \bar{A}}\right) \\
& =\frac{1}{1-u A-u \bar{A}-u w A \bar{A}} \\
& =\sum_{m \geq 0}(u A+u \bar{A}+u A w \bar{A})^{m} .
\end{aligned}
$$

The last formula is the same as (26). Therefore, $\mathrm{CF}_{\mathbf{L S}}$ is the generating function of $\mathcal{G}$ defined in (28), and the exponent of $y$ is the semi-length of all weakly flaw steps. We conclude the argument above by following properties.

Theorem 5.1 (a) The structure set $\mathcal{G}$ is a Chung-Feller extension of the little Schröder paths. (b) Let $\mathcal{A}_{n, m} \subseteq \mathcal{G}$ (respectively $\mathcal{B}_{n, m} \subseteq \mathcal{L S}_{\mathbb{N}}$ ) consist of those paths of length $2 n$ with $m$ steps $L_{2}$. Then we have $\left|\mathcal{A}_{n, m}\right|=(n+1)\left|\mathcal{B}_{n, m}\right|$. (c) Let $k \geq 1$. The (weighted respectively) $k$-colored $\mathcal{G}$ is a Chung-Feller extension of the (weighted respectively) $k$-colored little Schröder paths. (d) Given $\mathbf{m}=\left(m_{1}, \ldots, m_{k}\right)$ with each $m_{i} \in \mathbb{N}$. Let $\mathcal{A}_{n, \mathbf{m}} \subseteq \mathcal{G}$ (respectively $\mathcal{B}_{n, \mathbf{m}} \subseteq \mathcal{L S}_{\mathbb{N}}$ ) consist of 2 -paths that have $m_{i}$ steps $L_{2}$ colored by $i$. Then we have $\left|\mathcal{A}_{n, \mathbf{m}}\right|=(n+1)\left|\mathcal{B}_{n, \mathbf{m}}\right|$.

We demonstrate another $\mathrm{CF}$ extension of $\mathcal{L} \mathcal{S}_{n}$ by explaining

$$
\mathrm{CF}_{\mathbf{L S}}(x, 1,1, y)=\sum_{m \geq 0}(A+\bar{A}+A \bar{A})^{m}
$$

Note that $A=x G_{\mathcal{S C}}$ symbolizes lifted Schröder paths, i.e., paths of form $U$-a Schröder path- $D$. Similarly, $\bar{A}$ represents those paths of form $D$-a weakly flaw Schröder path- $U$. No doubt that $(A+\bar{A})^{m}$ represents concatenations of subpaths in these two forms. Let $\mathcal{K}_{n} \subseteq \mathcal{L}_{2 n, \mathcal{S C}}$ consist of the paths without prairie. The generating function of $\mathcal{K}_{\mathbb{N}}$ is exactly $\sum_{m \geq 0}(A+\bar{A})^{m}$. But how about $A \bar{A}$ ? We have to use a proper weight function to explain $\mathrm{CF}_{\mathbf{L S}}(x, 1,1, y)$. Given a path $P \in \mathcal{K}_{\mathbb{N}}$, let $\delta(P)$ be the number of consecutive $D$ - $D$ 's on $P$ that cross the $x$-axis, i.e., the number of $A \bar{A}$-type subpaths of $P$. Once an $A \bar{A}$-type subpath appears in $P$, at the same we shall consider this subpath as two components, namely $A$ and $\bar{A}$, and also consider it a single component, namely $A \bar{A}$. By this way we define a weight function as $\omega(P)=2^{\delta(P)}$ and then $\operatorname{CF}_{\mathbf{L S}}(x, 1,1, y)$ is equal to the generating function defined by

$$
\sum_{P \in \mathcal{K}_{\mathbb{N}}} \omega(P) x^{\ell(P) / 2} y^{f(P) / 2}
$$

where $f(P)$ is the total length of the flaw steps on $P$. 


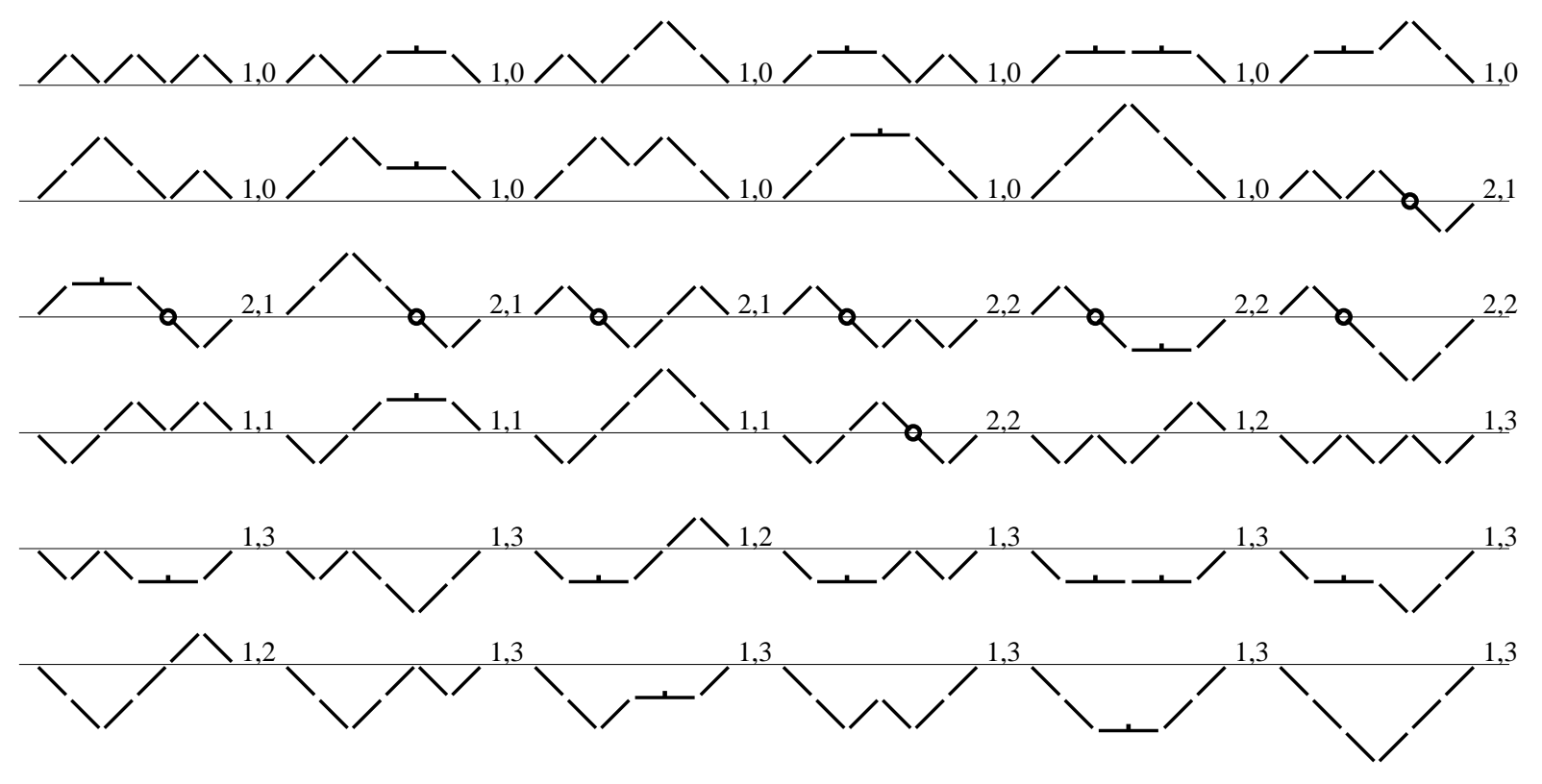

Figure 2: The $\omega$-weighted $\mathcal{K}_{3}$. The numbers are the weight and the semi-length of flaw steps.

Theorem 5.2 The $\omega$-weighted $\mathcal{K}_{n}$ is a Chung-Feller extension of the little Schröder $n$ paths.

We show Figure 2 to demonstrate $\omega$-weighted $\mathcal{K}_{3}$. Since $\left|\mathcal{L S}_{3}\right|=11$, the total weight of $\mathcal{K}_{3}$ is 44. Be aware of that the analogous properties as parts (b), (c) and (d) in Theorem 5.1 cannot apply here, because we plug $u=w=1$ into $\mathrm{CF}_{\mathbf{L S}}$ and then blur the distinction between steps $U$ and $L_{2}$.

\section{The final remark and the acknowledgements}

The ballot problem is a more general problem and draws our further interest. In a survey paper of Renault [22], four different proofs of the ballot theorem were reviewed, including the cycle lemma and the uniform partition method. Different from the previous bijective proofs, our study actually open a new branch for the uniform partition method by using generating functions. Several follow-up researches have been done by Huan, Ma and Yeh. Please, refer to $[14,19,20]$.

The authors would like to thank the anonymous referees for many valuable comments and suggestions. 


\section{References}

[1] D. André, Solution directe du problème résolu par M. Bertrand, Comptes Rendus de lÁcadémie des Sciences, Paris 105 (1887) 436-437.

[2] E. Barcucci, E. Pergola, R. Pinzani and S. Rinaldi, ECO Method and Hill-free Generalized Motzkin Paths, Sém. Lothar. Combin., 46 (2001), Article B46b.

[3] J. Bertrand, Solution d'un problème, Comptes Rendus de l'Académie des Sciences, Paris 105 (1887) 369.

[4] D. Callan, Pair them up!: A visual approach to the Chung-Feller Theorem, College Math. J., 26 (1995) 196-198.

[5] N. Cameron, The combinatorics of even trees, Congr. Numer., 147 (2000) 129-143.

[6] Y.-M. Chen, Note: The Chung-Feller theorem revisited, Discrete Math., 308 (2008) $1328-1329$.

[7] W.Y.C. Chen, S.H.F. Yan and L.L.M. Yang, Identities from weighted 2-Motzkin paths, Adv. Appl. Math., 41 (2008) 329-334.

[8] K.L. Chung and W. Feller, On fluctuations in coin-tossing, Proc. Nat. Acad. Sci. USA, 35 (1949) 605-608.

[9] N. Dershowitz and S. Zaks, The cycle lemma and some applications, European J. Combin., 11 (1990) 35-40.

[10] E. Deutsch, L.W. Shapiro, A bijection between ordered trees and 2-Motzkin paths and its many consequences, Discrete Math., 256 (2002) 655-670.

[11] S.-P. Eu, T.-S. Fu and Y.-N. Yeh, Refined Chung-Feller theorem for lattice paths, J. Combin. Theory Ser. A, 112 (2005) 143-162.

[12] S.-P. Eu, S.-C. Liu and Y.-N. Yeh, Taylor expensions for Catalan and Motzkin numbers, Adv. Appl. Math., 29 (2002) 345-357.

[13] P. Hilton and J. Pedersen, Catalan numbers, their generalization, and their uses, Math. Int., 13 (1991) 64-75.

[14] P.-Y. Huang, J. Ma, and Y.-N. Yeh, Cyclic permutations of sequences and uniform partitions, Electron. J. Combin., 17 (2010) \#R117

[15] D.A. Klarner, Correspondences Between Plane Trees and Binary Sequences. J. Combin. Theory, 9 (1970) 401-411.

[16] J. Labelle and Y.-N. Yeh, Dyck paths of knight moves, Discrete Appl. Math., 24 (1989) 213-221.

[17] J. Labelle and Y.-N. Yeh, Generalized Dyck paths, Discrete Math., 82 (1990) 1-6.

[18] P.A. MacMahon, Memoir on the theory of the partitions of numbers, Philos. Trans. Roy. Soc. London, Ser. A, 209 (1909), 153-175; Also G.E. Andrews (Ed.), Collected Works, vol. 1, MIT Press, Cambridge, MA, 1978, 1292-1314.

[19] J. Ma and Y.-N. Yeh, Generalizations of Chung-Feller theorem I, Bull. Inst. Math. Acad. Sin. (N.S.), 4 (2009), No. 3, 299-332. 
[20] J. Ma and Y.-N. Yeh, Refinements of $(n, m)$-Dyck paths, European J. Combin., 32 (2011) 92-99.

[21] T.V. Narayana, Cyclic permutation of lattice paths and the Chung-Feller theorem, Skand. Aktuarietidskrift, 50 (1967) 23-30.

[22] M. Renault, Four proofs of the Ballot Theorem, Math. Mag., 80 (2007) 345-352.

[23] M. Renault, Lost (and found) in translation: Andre's actual method and its application to the generalized Ballot Problem, Amer. Math. Monthly, 115 (2008) 358-363.

[24] D. G. Rogers and L.W. Shapiro, Some correspondences involving the Schröder numbers and relations, in Comb. Math., Proc. of the Intern. Conf., Canberra 1977, Lecture Notes in Mathematics 686, Springer-Verlag, 1978, 267-276.

[25] A. Sapounakis and P. Tsikouras, On k-colored Motzkin words, J. of Int. Seq., 7 (2004) Article 04.2.5.

[26] L.W. Shapiro, Some open questions about random walks, involutions, limiting distributions, and generating functions, Adv. Appl. Math., 27 (2001) 585-596.

[27] L.W. Shapiro and W.J. Woan, Some generating proofs of old and new results in probability, Congr. Numer., 143 (2000) 193-205.

[28] R.P. Stanley, Enumerative Combinatorics, Vol. 2, Cambridge University Press, Cambridge, 1999.

[29] R.P. Stanley, Catalan Addendum, http://www-math.mit.edu/ rstan/ec/catadd.pdf.

[30] J. West, Generating trees and the Catalan and Schröder numbers, Discrete Math., 146 (1995) 247-262.

[31] W.-J. Woan, Uniform partitions of lattice paths and Chung-Feller generalizations, Amer. Math. Monthly, 108 (2001) 556-559. 